\title{
SELF-SIMILAR VISCOUS GROWTH OF THE CENTRAL CORE OF AGN SEEDS
}

\author{
T. TSURIBE
}

Department of Astronomy, The University of Tokyo Bunkyo-ku, Tokyo 113

\section{Introduction}

Dynamical collapse of rotating cloud and subsequent mass supply to the central object is the basic physical process on the formation of the central black holes in the high-z QSO cosmologically. Considering the formation of super massive black holes, the centrifugal barrier plays an important role. In this contribution, I consider the viscous effect to the self-gravitating disk which formed cosmologically. In particular, unsteady growth of the central core is treated consistently with surrounding accreting disks semianalytically. A new self-similar solution of axisymmetric viscous accretion onto an evolving point mass is obtained. Application to QSO progenitor black hole formation is briefly discussed.

\section{Self-Similar Solutions}

In this work, Shu's (1977) isothermal 'inside-out' spherical self-similar solution is extended to the rotating self-gravitating disk with viscosity. Due to the rotation, inner asymptotic behavior is that of rotationally supported steady viscous disk instead of the free fall collapse. A series of solutions includes shock standing solution and globally rotationally supported solution without a shock. All physical quantities are characterized by two non-dimensional parameters $\alpha$ and $q$, where $\alpha$ specifies viscosity (Shakra \& Sunyaev 1973) and $q$ is proportional to the product of the initial ratio of thermal and rotation energy to gravitational energy. Mass accretion rate onto the central core is found to be constant $0.9 c_{\mathrm{s}}^{3} G^{-1} \alpha q^{-1}$ even if the non-steady evolution of the central core is considered. In inner region, there exists Keplerian disk solution $V_{r} \propto r^{1 / 2}, \Sigma \propto r^{-3 / 2}, \dot{M}=$ const. This 

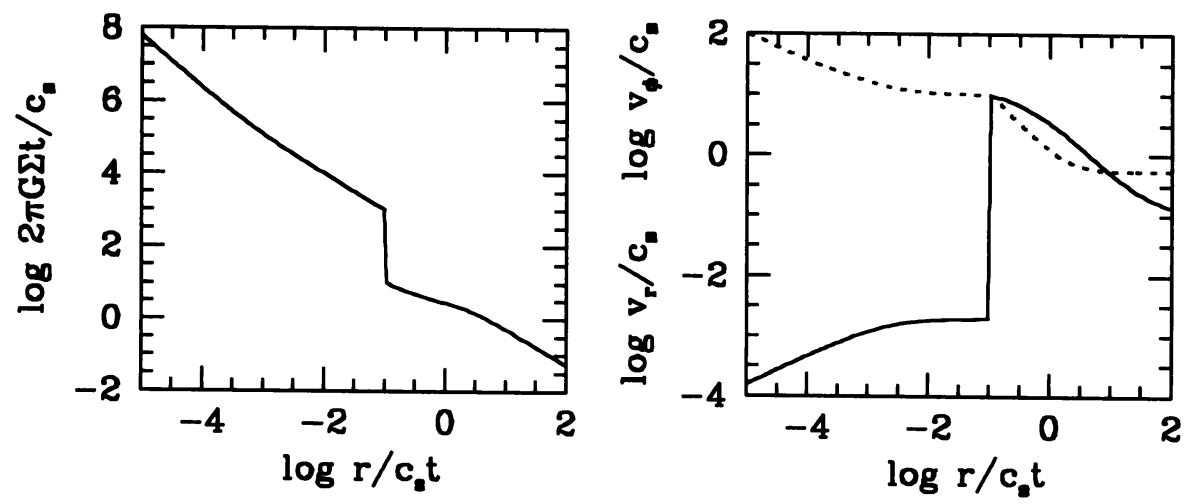

Figure 1. Self-similar solution with a shock

Keplerian region increases its outer radius with the growth of the central core as $r_{\mathrm{KD}}=2 \alpha q c_{\mathrm{s}} t$. Outer disk has profiles as $V_{r}=$ const., $\Sigma \propto r^{-1}$, and $\dot{M}=$ const. Outer boundary conditions determine whether shock stands or not. In figure 1, an example of self-similar solutions is shown. For a typical density fluctuation, mass accretion rate into the central core is

$$
\dot{M}=1.4\left(\frac{c_{\mathrm{s}}}{10 \mathrm{~km} / \mathrm{s}}\right)^{2}\left(\frac{\alpha}{0.1}\right)\left(\frac{R_{\mathrm{ta}}}{40 \mathrm{pc}}\right)^{-1 / 2}\left(\frac{M_{\mathrm{b}}}{10^{6} M_{\odot}}\right)\left(\frac{\lambda}{0.01}\right)^{-1} M_{\odot} / \mathrm{yr} .
$$

\section{Concluding Remarks}

The existence of the self-similar solution proposes that there is a possibility that the seed black holes can form as a result of the first collapse of the cosmological perturbations with low spin (Eisenstein \& Loeb 1995). Due to a viscosity, small central point mass can form and increases its mass linearly with time. As a result, massive black hole and the disk around the core form and grow in its mass and radius. Such a core with disk would be grows to become AGN central engine with sufficient mass supply from outer region by other fueling mechanism such as gravitational torque.

The author would like to thanks M. Umemura, S. Mineshige, J. Fukue and T. Nakamoto for useful discussions. This work is supported by JSPS Fellowships for Young Scientists. This work is done at the Center for Computational Physics, University of Tsukuba.

\section{References}

Shakura, N. I. \& Sunyaev, R. A. 1973, A\&Ap, 24, 337

Shu, F. H. 1977, ApJ, 214, 488

Eisenstein, D. J. \& Loeb, A. 1994, ApJ, 1995, 443, 11 